\title{
Unzipping Dynamics of Long DNAs
}

\author{
Simona Cocco ${ }^{1}$, Rémi Monasson ${ }^{2,3}$ and John F. Marko ${ }^{4}$ \\ ${ }^{1}$ Laboratoire de Dynamique des Fluides Complexes, 3 rue de l'Université, 67000 Strasbourg, France. \\ 2 Laboratoire de Physique Théorique de l'ENS, 24 rue Lhomond, 75005 Paris, France. \\ ${ }^{3}$ Laboratoire de Physique Théorique, 3 rue de l'Université, 67000 Strasbourg, France. \\ ${ }^{4}$ University of Illinois at Chicago, Department of Physics, 845 West Taylor Street, Chicago IL 60607-7059.
}

\begin{abstract}
The two strands of the DNA double helix can be 'unzipped' by application of $\approx 15 \mathrm{pN}$ force. We analyze the dynamics of unzipping and rezipping, for the case where the molecule ends are separated and re-approached at constant velocity. For unzipping of 50 kilobase DNAs at less than about 1000 bases per second, thermal equilibrium-based theory applies. However, for higher unzipping velocities, rotational viscous drag creates a buildup of elastic torque to levels above $k_{B} T$ in the dsDNA region, causing the unzipping force to be well above or well below the equilibrium unzipping force during respectively unzipping and rezipping, in accord with recent experimental results of Thomen et al. [Phys. Rev. Lett. 88, 248102 (2002)]. Our analysis includes the effect of sequence on unzipping and rezipping, and the transient delay in buildup of the unzipping force due to the approach to the steady state.
\end{abstract}

\section{INTRODUCTION}

Double stranded DNA (dsDNA, the DNA double helix) is the genetic memory element of all cells. Two copies of the genetic information are encoded into the two complementary-sequence strands, which are base-paired together through most of the cell cycle. However, the two strands must be completely separated during DNA replication, and partially separated during DNA transcription. In cells, the separation of DNA strands occurs via forces applied by DNA-processing machinery. Force-driven dsDNA 'unzipping' is therefore of direct biological relevance.

A few groups have carried out single-molecule studies of DNA unzipping by force. Essevaz-Roulet, Bockelmann and Heslot [1,2] have studied the $\approx 15 \mathrm{pN}$ forces encountered during unzipping of $50 \mathrm{~kb} \lambda$ DNA. Variations of the unzipping force with sequence were observed, which are over the range of about 10 to $20 \mathrm{pN}$. Other groups have carried out similar experiments on unzipping of DNA [3, 4 ] and on RNA helix-loop structures [5], observing similar unzipping forces.

A number of theoretical works [6 13] have addressed the equilibrium statistical mechanics of dsDNA unzipping, with particular emphasis on the effects of sequence. Unzipping driven by DNA torque ('DNA unwinding') has drawn much less attention in spite of elegant experiments [14] and corroborating theory [15]. As a result, theoretical consideration of the combined effects of force and torque on unzipping have only recently been discussed [12]. Since DNA unzipping involves rotation of the remaining double-helical DNA (Fig. 1), one expects that rotational drag torque should produce a contribution to the force needed to unzip DNA. Experimentally, no dependence of force on the rate of unzipping up to about 1000 base pairs per second (bp/s) has been observed. However, in recent experiments of Heslot an appreciable increase (up to 40\%) in unzipping force was observed, at unzipping rates in the range of $10 \mathrm{kbp} / \mathrm{s}$ [16].

This paper presents theoretical analysis of the velocity dependence of the unzipping force, for large molecules where the kinetics is dominated by viscous effects. To do this we introduce a dynamic model of unzipping which combines the polymer stretching dynamics of the extending single-stranded DNA (ssDNA) regions, the rotation of the remaining double-stranded (dsDNA) region due to the unwinding generated by the unzipping [17 [19], and the kinetics of the translation of the 'fork' separating the ssDNA and dsDNA regions. Although some work has been done on the Langevin dynamics of DNA unzipped by constant tension [8, 10, 12], a number of open questions remain. These include the unzipping force for constant end-to-end displacement velocity, the effect of sequence, and the role of rotation of the dsDNA.

Below, kinetic equations for unzipping show that beyond a certain unzipping rate, the predictions of the theory of equilibrium unzipping cease to apply. For a $50 \mathrm{~kb}$ dsDNA this critical rate is about $1000 \mathrm{bp} / \mathrm{s}$, similar to the threshold seen experimentally [16]. In our theory there is a buildup of elastic torque in the dsDNA due to the drag torque associated with the dsDNA rotation. The ssDNA stretching degrees of freedom and the fork region itself remain near to equilibrium at experimentally accessible unzipping rates. We show that the relation between the unzipping force and the elastic torque buildup during opening is simply determined by the equilibrium coexistence between closed and opened and stretched base pairs, described by the binding-unbinding force-torque 'phase diagram'.

We first describe the experimental situation in Section II, and then in Section III we review the equilibrium theory of DNA unzipping, using a mean-field approach. We discuss the effects of force applied to the ssDNA ends, and torque applied 'upstream' to the dsDNA for homogeneous and heterogeneous sequences. In Sec. IV we discuss the 
relaxation of ssDNA stretching and dsDNA twisting, and we then present a dynamic model for the propagation of the ssDNA-dsDNA 'fork' region for homogeneous and heterogeneous sequences. We first present a simple theory where we assume that the dsDNA twist is in a steady state. The problem of rezipping of a dsDNA is also considered, and it is shown that for rapid retraction, a left-handed viscous torque delays recombination of the ssDNAs. Finally in Sec. $\mathrm{V}$ we analyze the DNA twist dynamics in order to understand the delay in force buildup observed experimentally at high unzipping rates [16].

\section{EXPERIMENTAL SETUP}

The experiment of Thomen, Bockelmann and Heslot [16] unzips a $\lambda$ dsDNA of 48502 base pairs (bp) in $10 \mathrm{mM}$ phosphate buffer, $150 \mathrm{mM} \mathrm{NaCl}, \mathrm{Ph} 7$, at room temperature. The $\lambda$ dsDNA is attached to two dsDNA linker arms each of $7000 \mathrm{bp}$; one of these is anchored to a movable glass slide, and the other is attached to a $\approx$-micron-diameter silica bead which is held in a laser trap (Fig. 1). The displacement of the glass slide at a controlled velocity in the range 1 to $20 \mu \mathrm{m} / \mathrm{s}$ forces the molecule to open. After the molecule is opened (typically after $\approx 25 \mu \mathrm{m}$ of displacement) the stage motion is inverted, allowing the molecule to rezip into dsDNA (reannealing). The force transmitted to the ends of the linkers during the experiment is measured using the position of the bead in the laser trap, which is of stiffness $k_{\text {opt }} \approx 0.25 \mathrm{pN} / \mathrm{nm}$.

The stiffnesses of the two dsDNA linkers and the two ssDNAs depend on force, but are known from other experiments on dsDNA and ssDNA. At forces near $15 \mathrm{pN}$ the total stiffnesses of the two dsDNAs (together 14000 base pairs) is $k_{d s} \approx 0.1 \mathrm{pN} / \mathrm{nm}$; the stiffness of the two ssDNAs depends on the number of unzipped base pairs $n$, and is $k_{s s} \approx 70 / n \mathrm{pN} / \mathrm{nm}$. Below, we will neglect the $\approx 0.1 \mu \mathrm{m}$ shift of the bead in the laser trap.

\section{STATIC UNZIPPING}

The basic unzipping force follows from the simple argument that unzipping will be thermodynamically favorable when the free energy required for unzipping one base, $1 \leq g \leq 4 k_{B} T$, is equal to the mechanical work done, $f \ell$, where $\ell \approx 1 \mathrm{~nm}$ is the projected length of ssDNA liberated during unzipping of one base pair. The resulting force $f \approx g / \ell$ is on the order of $10 \mathrm{pN}$ as it has been observed experimentally. Below we describe static unzipping in more detail, focusing on the force and number of opened base pairs at fixed displacement. The equilibrium theory of unzipping describes experiments for velocities small enough to allow the system to stay in thermal equilibrium.

\section{A. Homogeneous sequence}

We start by considering an idealized homogeneous sequence, with a uniform $g_{0}=2.5 k_{B} T$, the averaged value on the $\lambda$ sequence. The free energy cost $g$ per opened base pair may be obtained for the $\lambda$ sequence, for the experimental conditions described above, using the Mfold program [20] with stacking and pairing free energies measured by Santa Lucia [21].

To describe unzipping of the dsDNA we write down the work that must be done by the force to separate the ends of the linkers by a distance $2 x$. This includes the work done by the force to extend the two dsDNA linker arms by $2 x_{d s}$, the free energy cost of opening $n$ base pairs of the $\lambda$ DNA, and the work done by the force to keep the ends of the ssDNA regions separated by $2\left(x-x_{d s}\right)$, and is therefore a function of the number $n$ of opened base pairs, the extension $2 x_{d s}$ of the dsDNA linker arms, and the total end-to-end distance $2 x$. We also include the work done by torque $\Gamma$ applied to the end of the dsDNA region; this will be essential to considering the drag opposing rapid rotation of the dsDNA region. This free energy reads:

$$
F_{x}\left(n, x_{d s}\right)=2 W_{d s}\left(x_{d s}\right)+2 W_{s s}\left(x-x_{d s}, n\right)+n\left[g_{0}+\Gamma \theta_{0}\right]
$$

Here $W(x)=\int_{0}^{x} f\left(x^{\prime}\right) d x^{\prime}$ is the work done by the stretching force $f(x)$ at fixed extension, for the dsDNA linker arms and the unzipped ssDNA, and $\theta_{0}=2 \pi / 10.5=0.60$ is the number of radians of rotation made during opening of each base pair; this model is discussed in more detail in Ref. [12].

In the range of forces of 10-30 pN the dsDNA is extended enough that the leading contribution from its high-force entropic elasticity, plus linear stretching elasticity, give an accurate model:

$$
x_{d s}(f)=L_{d s}\left[1-\frac{1}{2}\left(\frac{k_{B} T}{f A}\right)^{\frac{1}{2}}+\frac{f}{\gamma_{d s}}\right]
$$


We use persistence length $A=48 \mathrm{~nm}$ and Young modulus $\gamma_{d s}=1000 \mathrm{pN}$ as determined by separate experiments [22]. The length of each $7 \mathrm{kbp}$ linker is $L_{d s}=2.38 \mu \mathrm{m}$.

The ssDNA is described by the freely-jointed-chain-like (FJCL) model [23], which gives the extension of $n$ of the unzipped bases as:

$$
x_{s s}=n l_{s s}(f)
$$

where

$$
l_{s s}(f)=d\left[\operatorname{coth}\left(\frac{f b}{k_{B} T}\right)-\frac{k_{B} T}{f b}\right]\left[1+\frac{f}{\gamma_{s s}}\right]
$$

The monomer length $d=0.56 \mathrm{~nm}$, the segment length $b=1.4 \mathrm{~nm}$, and the stretching elastic constant $\gamma_{s s}=800 \mathrm{pN}$ are fitted from experimental data [23]. Eqs. (2) and (4) can be used to obtain free energies via integration by parts:

$$
W(x)=x f(x)-\int_{0}^{f(x)} x\left(f^{\prime}\right) d f^{\prime} .
$$

Given the $W$ 's and the total half-extension $x$, the minimum of the total free energy with respect to linker arm extension $x_{d s}, \partial F_{x}\left(n, x_{d s}\right) / \partial x_{d s}=0$, equilibrates ssDNA and linker tensions. Then, minimization of the free energy with respect to the number of opened base pairs $n, \partial F_{x}\left(n, x_{d s}\right) / \partial n=0$, determines the equilibrium unzipping force $f_{u}$, via:

$$
2 w_{s s}\left(f_{u}\right)-\Gamma \theta_{0}=g_{0}
$$

where $w_{s s}(f) \equiv \int_{0}^{f} l_{s s}\left(f^{\prime}\right) d f^{\prime}$.

Eq. (6) is a first-order transition coexistence condition, stating that work done by the force and torque in opening a base pair equals the base pairing free energy. Fig. 2 shows the solution of (6) plotted in the torque-force plane. Note that overwinding torque in the dsDNA $(\Gamma>0)$ increases the unzipping force. The size of dsDNA torque needed to appreciably shift up $f_{u}$ is $g_{0} / \theta_{0} \approx 4.2 k_{B} T$; a left-handed (negative, corresponding to dsDNA unwinding) torque of this magnitude makes unzipping occur for zero force, close to the unwinding torque inferred from experiments [14]. Below we will calculate how the overwinding transiently built up in the dsDNA during rapid unzipping, resulting from rotational friction upstream of the unzipping 'fork', will boost the ssDNA tension.

The number of unzipped base pairs is simply calculated from the condition that the total displacement $2 x$ is equal to the extension of the two linkers arms $2 x_{d s}$ plus the extension of the two single unzipped single strands $2 x_{s s}$ :

$$
x=n l_{s s}(f)+x_{d s}(f)
$$

At the beginning of an unzipping experiment, the two linker arms first stretch until the extension $x_{d s u} \equiv x_{d s}\left(f_{u}\right)$ is reached. At this point the $\lambda$ DNA starts to unzip, and the force stays pegged at $f_{u}$, with the number of opened base pairs proportional to further displacement:

$$
n_{u}(x)=\frac{x-x_{d s}\left(f_{u}\right)}{l_{s s}\left(f_{u}\right)}
$$

The equilibrium force and number of unzipped base pairs for this homogeneous model, with $g_{0}=2.5 k_{B} T$ and at zero torque, are plotted in Fig. 3. The critical unzipping force is $f_{u} \approx 16 \mathrm{pN}$, and the average projection of each unzipped base along the unzipping direction is $l_{s s}\left(f_{u}\right)=0.44 \mathrm{~nm}$. This is close to what is observed experimentally [16] at small displacement velocities $v \leq 1 \mu \mathrm{m} / \mathrm{s}$; as unzipping proceeds the ssDNA is stretched to about $50 \%$ of its total contour length of $\approx 1 \mathrm{~nm} /$ base.

Given accurate knowledge of the elasticity of ssDNA, unzipping experiments can determine the pairing free energy at room temperature. For the homogeneous model, we find $g_{0}=2.5 k_{B} T$. Prior to these experiments, this free energy difference was indirectly inferred from model free energies obtained from study of DNA melting at temperatures of 20 to 40 degrees above room temperature [24]. A sequence-averaged point of view as presented above can give a rough account of thermodynamics of unzipping of large molecules. However, there is appreciable sequence-dependence of the base-pairing free energy. Rief et al have found that pure AT (the most loosely bound base pairs) sequences unzip at about $9 \mathrm{pN}$, while pure GC sequences (the most tightly bound base pairs) open at about $20 \mathrm{pN}$ [3]. This range of force corresponds to base-pairing free energies of 0.8 to $3.8 k_{B} T$ per base pair. Therefore, a more detailed analysis of unzipping, and especially describing unzipping of short inhomogeneous sequences, requires models which take into account sequence-dependence and the cooperativity of strand separation [24]. 


\section{B. Heterogeneous sequence}

Sequence effects can be added by making $g_{0}$ a function of $n$. The equilibrium opening of $\lambda$-DNA at zero torque has been theoretically analyzed by Bockelmann, Essevaz-Roulet and Heslot [2], who have numerically calculated the thermal average of the force and the number of opened base pairs. They included thermal fluctuations of the ssDNA and dsDNA regions using a free energy of the form (11) plus trap/cantilever elastic energy. Here we show how to obtain essentially the same results, using a preaveraging of $g_{0}(n)$.

The approach of the previous section computes the free energy of a given number $n$ of opened base pairs, at fixed displacement $x$, using (1) and (耳). We estimate the fluctuations of the ssDNA, the dsDNA and the laser trap/cantilever, at $f_{u} \approx 15 \mathrm{pN}$ using their combined stiffness [25] $k_{t o t}=\left[1 / k_{s s}+1 / k_{d s}+1 / k_{o p t}\right]^{-1}$. This net stiffness decreases with the opening because of the inverse proportionality of $k_{s s}$ to the number of opened base pairs. During opening of the first $5 \mathrm{kbp}$ of $\lambda$, the extension fluctuations in the length are $\approx 20 \mathrm{~nm}$, corresponding to 20 base pairs.

To account for these fluctuations we have Gaussian-preaveraged the denaturation free energy $g(n)$ using a standard deviation of 10 base pairs. To each configuration of $n$ opened base pairs is associated a Boltzmann factor using free energy (17) where the force is determined by the condition (77). The thermal averages of the number of unzipped base pairs and of the force, as a function of the displacement $2 x$, are plotted in Fig. 4 , for displacement after opening up to $5 \mu \mathrm{m}(\approx 5 \mathrm{kbp})$.

The result of this calculation is in good agreement with experiment and the computation of Bockelmann et al. [25]. The average slope of the opening curve gives the average extension of each unzipped segment along the unzipping direction $2 l_{s s}=0.95 \mathrm{~nm}$ (one base pairs opens for each $0.95 \mathrm{~nm}$ of displacement). As in the experiment, the sequence generate a stick-slip motion; the opening fork stalls at G-C-rich parts of the sequence, giving a sawtooth pattern in the force signal and a step pattern in the number of opened base pairs [25, 10].

\section{UNZIPPING DYNAMICS}

To describe the motion of the unzipping fork, we must consider four physical effects. First and second, we must consider the elongating dynamics of the dsDNA linker arms, and the unzipped ssDNA. Third, we must worry about the propagation of twist down the dsDNA; each 10.5 bases unzipped forces one more full right-handed twist into the unzipped dsDNA region. Finally, we must close the dynamical equations with a model for the translation of the ssDNA-dsDNA fork in $n$. For each of these processes we consider relaxational dynamics of the form:

$$
\zeta \frac{\partial z}{\partial t}=-\frac{\partial F}{\partial z}
$$

Here $z$ can be the position of a monomer of ssDNA or of dsDNA, the twist orientation $\theta$ of a monomer of the dsDNA being opened, or the number of unzipped base pairs $n$. In each case $F$ is the free energy, while $\zeta$ is the relevant friction constant. For translational motion of ssDNA or dsDNA monomers, $\zeta \equiv 6 \pi \eta a$; we take $a=1 \mathrm{~nm}$. For twist relaxation, $\zeta \equiv 4 \pi \eta r^{2}$ where $r$ is the dsDNA hydrodynamic radius, and where $\eta=0.001 \mathrm{~Pa} \cdot \mathrm{s}$, the suitable value for aqueous buffer. We use $r=2 \mathrm{~nm}$, twice the 'bare' chemical radius of the double helix; this generates force curves in accord with the experiment [16]. Note that $r$ is the only adjustable parameter of our theory; it is essentially a friction constant.

\section{A. DNA stretch and twist relaxation times}

Since ssDNA and dsDNA are appreciably stretched by the $>10 \mathrm{pN}$ forces applied during unzipping, their dynamics is reasonably described by local hydrodynamic friction. Logarithmic corrections due to long-range hydrodynamic coupling can be added to this discussion but without major effect. To estimate the order of magnitude of the dsDNA linkers and the ssDNA to equilibrate, we expand the stretching free energies around the force of $f=15 \mathrm{pN}$ (the nonlinear elasticity of the previous section is used) to obtain:

$$
F=\frac{K}{2} \sum_{n}\left[z(n, t)-z(n-1, t)-z_{0}\right]^{2}
$$

where $z(n, t)$ is the position along the unzipping direction of either the $n$th monomer of the dsDNA, or of the unzipped ssDNA. In these two cases the monomer stiffness are either $K_{d s}=1400 \mathrm{pN} / \mathrm{nm}(\mathrm{dsDNA})$ or $K_{s s}=140 \mathrm{pN} / \mathrm{nm}$ (ssDNA). Note that these should note be confused with the polymer stiffnesses $k_{d s}$ and $k_{s s}$ discussed previously. 
The dsDNA twisting free energy is well described by the free energy of an elastic rod [26] in the range of torques relevant to unzipping experiments. This free energy may be written as in (10) where the degree of freedom $z(n, t)$ is the twist angle of base pair $n$. The twist stiffness is $K \equiv k_{B} T C / \Delta^{2}$ where the base rise $\Delta=0.34 \mathrm{~nm}$ converts base index $n$ to dsDNA contour length (note $k_{B} T C=80 \pm 20 \mathrm{~nm}$ is the usual elastic-rod twist rigidity [26])

The longest relaxation time of (9) with an elastic free energy (10) is

$$
t=\frac{\zeta \mathcal{N}^{2}}{k \pi^{2}}
$$

where either $\mathcal{N} \equiv N_{d s}=14000$ is the number of base pairs in the two linkers DNA, the number of unzipped base pairs $\mathcal{N} \equiv 2 n$, or the number of still zipped base pairs $\mathcal{N} \equiv N-n$. Substituting the relevant stiffness $k$ and $\operatorname{drag} \zeta$ (11), we obtain $t_{d s}=3 \cdot 10^{-4} \mathrm{~s}$ for dsDNA stretch relaxation, $t_{s s}=(2 n)^{2} \times 1.4 \cdot 10^{-11} \mathrm{~s}$ for ssDNA stretch relaxation, $t_{t w}=(N-n)^{2} \times 2 \cdot 10^{-12} \mathrm{~s}$ for dsDNA twist relaxation times.

Equilibrium will be reached for stretching or twisting if the relevant relaxation time is less than the unzipping time $1 \mathrm{~nm} \times t_{u} \approx n / v$. For the maximum velocities we are considering, $2 v=20 \mu \mathrm{m} / \mathrm{s}, t_{u}=n \times 5 \cdot 10^{-5} \mathrm{~s}$. Since $t_{s s} / t_{u} \approx n / 10^{6}$, ssDNA in unzipping experiments where $2 v \leq 20 \mu \mathrm{m} / \mathrm{s}$ will be equilibrated until about $10^{6}$ bp are unzipped. Therefore dsDNA and ssDNA stretching is at equilibrium in experiments on $\lambda$-DNA $\left(n<5 \times 10^{4}\right)$.

By contrast, twist relaxation cannot reach equilibrium at the start of unzipping; the relaxation time at $n=0$ is $t_{0}=5 \cdot 10^{-3} \mathrm{~s}$. Thus, we will now describe the fork dynamics, treating the ssDNA and dsDNA stretching in equilibrium. We first develop a theory of steady-state twisting; in Sec. V we analyze the approach to this steady state.

\section{B. Fork motion}

The fork position $n(t)$ will change as a result of the imbalance of ssDNA tension, opening cost, and dsDNA torque. Unzipping experiments occur at less than $20 \mathrm{~kb} / \mathrm{s}$, slow enough that each base is opened on average slower than single-base opening-closing times which are less than a microsecond. A quasistatic model of fork motion is plausible, with fork velocity in proportion to the free energy change associated with unzipping of one base.

We write the relaxation equation (9) for the fork motion in the continuum limit for the number of opened base pairs:

$$
\tau_{n} \frac{d n}{d t}=\frac{1}{k_{B} T}\left(2 w_{s s}(f)-g_{0}(n)-\Gamma \theta_{0}\right)
$$

The single-base relaxation time $\tau_{n}$ should be on the order of the diffusion time for the $\approx$ nm-long bases $\tau_{n} \approx$ $6 \pi \eta a^{3} / k_{B} T \approx 10^{-8} \mathrm{~s}$. The force $f(n, x)$ is again determined from (耳), and is implicitly a function of both the displacement and the number of open bases.

Equation (12) indicates that if the ssDNA tension is large, the fork moves to larger $n$. The fork is static $(d n / d t=0)$ for the equilibrium state (6). Each unzipped base forces the upstream dsDNA to rotate through $\theta_{0}=0.63 \mathrm{rad}$. If this fork rotation is sufficiently rapid, the viscous rotational drag along the $N-n$ dsDNA base pairs which remain to be unzipped, will generate elastic torque.

In this subsection we assume that the DNA to be unzipped has reached a stationary state rotating at a uniform angular velocity $\omega$. Since each opened base forces a rotation of the dsDNA region by an angle $\theta_{0}$, we have $\omega=\theta_{0} d n / d t$. The viscous torsional drag for the dsDNA, treated as a cylinder of cross-sectional radius $r$ and length $\Delta(N-n)$, is:

$$
\Gamma(n)=4 \pi \eta r^{2} \Delta(N-n) \omega
$$

Combining (13) and (12) we obtain the equation of motion for the fork position:

$$
\frac{d n}{d t}=\frac{1}{k_{B} T} \frac{2 w_{s s}(f)-g_{0}(n)}{\tau_{n}+(N-n) \tau_{r}}
$$

where

$$
\tau_{r}=\frac{4 \pi \eta r^{2} \Delta \theta_{0}^{2}}{k_{B} T}=2 \cdot 10^{-9} \mathrm{~s}
$$

The time $\tau_{r}$ is comparable to the value expected for $\tau_{n}$ (both are viscous times at the nanometer scale), but since $\tau_{r}$ appears in (14) magnified by a factor $\approx N$ relative to $\tau_{n}$, the rotational dynamics will be rate-limiting in most experimental situations. Also note that Eq. (14), and $\tau_{r}$ are independent of the value of the twist elastic constant $C$. Numerical integration of (14) gives force and torque during unzipping. The initial condition is that unzipping begins when the force in the linkers reaches $f_{u}$, i.e. $n\left(t=x_{d s u} / v\right)=0$. 


\section{Rezipping dynamics}

In the experiment of [16], following unzipping of $\approx 25000$ base pairs the molecule is allowed to rezip ('reanneal' in the nomenclature of biochemistry), by reversing the direction of the pulling velocity. If velocity is made negative, (14) describes this process. During rezipping, the dsDNA rotates in a right-handed sense, generating a left-handed drag torque on the molecule.

As shown in the phase diagram of Fig. 2 a negative torque promotes helix opening, decreasing the unzipping force. Therefore, the force during rezipping at high velocities is lower than the equilibrium unzipping force $f_{u}$; the force drops progressively during rezipping because the rotational drag (13) increases with dsDNA length. Force during retraction is calculated via integration of (14) (with $v \rightarrow-v$ ), starting from initial condition $n=25000$.

\section{Analytical estimate of unzipping force dependence on velocity}

We can estimate the increase of steady-state unzipping force with velocity using (14) for homogeneous sequence $g_{0}=2.5 k_{B} T$. Changing variables from $t$ to $x=v t$, (14) can be rewritten as,

$$
\frac{d n}{d x}=\frac{1}{\left(k_{B} T\right) v}\left[\frac{2 w_{s s}(f)-g_{0}}{\tau_{n}+(N-n) \tau_{r}}\right]
$$

where $f(x, n)$ is the equilibrium tension given by (76). In the limit of slow unzipping, the square bracket of (16) goes to zero, giving the equilibrium unzipping force $f_{u}$ (6) and the relationship (8) between extension $x$ and number $n_{u}$ of unzipped base pairs, $n_{u}(x)=\left(x-x_{d s u}\right) / l_{s s}\left(f_{u}\right)$.

We now suppose that the velocity is small (in a sense made more precise in the following), and that the unzipping force $f$ and number $n$ of unzipped base pairs can be expanded to first order in $v$, i.e. $f(x)=f_{u}+f_{1}(x) v+O\left(v^{2}\right)$ and $n(x)=n_{u}(x)+n_{1}(x) v+O\left(v^{2}\right)$. Plugging this into (6) and (16) permits us to obtain two coupled equations involving $f_{1}$ and $n_{1}$, with the results

$$
\begin{aligned}
& f_{1}(x)=k_{B} T\left(\frac{\tau_{n}+\left[N-n_{u}(x)\right] \tau_{r}}{2\left[l_{s s}\left(f_{u}\right)\right]^{2}}\right), \\
& n_{1}(x)=-\left[x_{d s}^{\prime}\left(f_{u}\right)+l_{s s}^{\prime}\left(f_{u}\right) n_{u}(x)\right] \frac{f_{1}(x)}{l_{s s}\left(f_{u}\right)},
\end{aligned}
$$

where $h^{\prime}$ denotes the derivative of $h$ with respect to its argument.

Therefore, at low velocity, the unzipping force reads

$$
f(x)=f_{u}\left(1+\frac{v}{v^{*}(x)}+O\left(v^{2}\right)\right)
$$

where

$$
v^{*}(x)=\frac{2 f_{u} l_{s s}\left(f_{u}\right)^{2}}{k_{B} T\left[\left(N-n_{u}(x)\right) \tau_{r}+\tau_{n}\right]} .
$$

The function $v^{*}(x)$ is plotted in the inset of Fig. 5. It is a rapidly increasing function of displacement $x$, bounded from below by $v^{*}\left(x_{d s u}^{+}\right) \approx 20 \mu \mathrm{m} / \mathrm{s}$ for $\lambda$-DNA $\left(N=5 \times 10^{4} \mathrm{bp}\right)$. Therefore, one can expect to observe a large increase in the initial unzipping force for velocities larger than a few $\mu \mathrm{m} / \mathrm{s}$.

Fig. 5 shows this force vs. velocity behavior which should be an upper bound to forces observed during the unzipping of a $\lambda$-DNA (dashed line). The theory indicates a $10 \%$ increase in unzipping force as $v$ is increased to about $2 \mu \mathrm{m} / \mathrm{s}$, comparable to the initial rate of increase recently observed [16] (velocities reported by Thomen et al. correspond to $2 v$, see Fig. 1).

Eqs. (18) and (19) also show that as the molecule unzips and $N-n$ goes down, the torsional drag on the dsDNA is reduced, and the force needed to keep the fork moving goes down. For low velocity, this force drop will be a nearly linear function of $N-n_{u}$. This is the signature that the twist transport dominates the fork retardation. Finally, we note that (18) can be used to estimate how the force depends on velocity during retraction by simply inverting the sign of the velocity. 


\section{E. Rezipping at zero tension}

For sufficiently fast retraction the reannealing of the double helix, the rate of which will be limited by the rotational drag on the dsDNA region, will not be able to keep up with the retraction. As a result the force should essentially drop to zero. This effect will be especially pronounced during the later stages of rezipping, since the rotational drag experienced by the dsDNA region increases as rezipping proceeds.

The velocity for 'free' rezipping under zero tension can be easily estimated, using the equation of motion (14) for the dsDNA-ssDNA fork:

$$
\frac{d n}{d t}=-\frac{1}{k_{B} T} \frac{g_{0}}{\tau_{n}+(N-n) \tau_{r}}
$$

Taking initial condition $n(t=0)=n_{0}$, we obtain

$$
n(t)=N+\frac{\tau_{r}}{\tau_{n}}-\left[\left(N+\frac{\tau_{n}}{\tau_{r}}-n_{0}\right)^{2}+2 \frac{g_{0}}{k_{B} T} \frac{t}{\tau_{r}}\right]^{1 / 2}
$$

Recall that $\tau_{n} / \tau_{r}=5$ is not a large number. Therefore, for long $\left(N>10^{3}\right)$ and initially totally open $\left(n_{0}=N\right)$ molecules, the rezipping follows $N-n \approx\left(2 g_{0} t /\left(k_{B} T \tau_{r}\right)\right)^{1 / 2}$, where the exponent is determined by the linear dependence of the total rotational drag on the size of the rezipped domain.

The total time needed to rezip for a 'free' fork is therefore $T \approx\left(2 N-n_{0}\right) n_{0} \tau_{r} k_{B} T /\left(2 g_{0}\right)$, which for $n(0)=25000$ is $\approx 0.75 \mathrm{~s}$. Therefore, for half-unzipped $\lambda$-DNA, retraction velocities of $2 v \geq 50 \mu \mathrm{m} / \mathrm{s}$ are at essentially zero tension. Equations (13) and (20) lead to the dsDNA torque for zero-tension rezipping:

$$
\Gamma=-\frac{g_{0}}{\theta_{0}}\left[1+\frac{\tau_{r}}{\tau_{n}(N-n(t))}\right]^{-1},
$$

with $n(t)$ given by (21). The maximum torque that appears at the fork during zero-force rezipping is the critical torque for the opening at zero force $g_{0} / \theta_{0}$, in accord with the phase diagram of Fig. 2 .

\section{F. Results for homogeneous sequence}

We now present numerical results for integration of (14) for the homogeneous case $g_{0}=2.5 k_{B} T, \eta=10^{-3} \mathrm{~Pa}$, $r=2 \mathrm{~nm}, \Delta=0.34 \mathrm{~nm}$, and $\theta_{0}=2 \pi / 10$. The ssDNA elasticity FJCL parameters are $b=1.4 \mathrm{~nm}, d=0.56 \mathrm{~nm}$ and $\gamma_{s s}=800 \mathrm{pN}$. The dsDNA linker elasticity parameters are $L_{d s}=2.38 \mu \mathrm{m}, A=41 \mathrm{~nm}$ and $\gamma_{d s}=1300 \mathrm{pN}$. The fork and torsional relaxation times are $\tau_{n}=10^{-8} \mathrm{~s}, \tau_{r}=210^{-9} \mathrm{~s}$.

Force as a function of displacement after opening is shown in Fig. 6 for velocities $2 v$ of $4,8,16$ and $20 \mu \mathrm{m} / \mathrm{s}$. During unzipping one observes an initial force upswing as the dsDNA linkers are first tensed, followed by a force peak and a gradual force reduction due to the decrease of torsional drag, as unzipping proceeds. For $2 v=20 \mu \mathrm{m} / \mathrm{s}$ the peak force is $f_{\text {max }} \simeq 23 \mathrm{pN}$, corresponding to a $7 \mathrm{pN}$ unzipping force increase relative to the equilibrium value $f_{u}=15.7 \mathrm{pN}$; this is in good agreement with the increase of $\approx 10 \mathrm{pN}$ observed in the experiment of [16]. On the other hand, the initial force increase observed experimentally is smoother than the theory of Fig. 6. Below we will show how sequence effects and twist relaxation dynamics reduce the initial rate of force increase.

Fig. 6 also shows force during retraction. For $2 v=-4 \mu \mathrm{m} / \mathrm{s}$, there is already a noticeable force hysteresis relative to the $2 v=+4 \mu \mathrm{m} / \mathrm{s}$ extension curve. For $2 v \geq-8 \mu \mathrm{m} / \mathrm{s}$, the ssDNA force approaches zero at the end of the retraction cycle. Similar 'hysteresis loops' were observed by Thomen et al. [16.

Fig. 7 shows the DNA torque at the unzipping fork, during these unzipping-rezipping cycles. The unzipping torque reaches a peak coincident with the force peaks of Fig. 6. At $2 v=20 \mu \mathrm{m} / \mathrm{s}$ the maximum torque during unzipping is $\Gamma_{\max }=2.8 k_{B} T$, while the maximum unwinding torque during rezipping is $\Gamma_{\max }=-3.8 k_{B} T$, slightly smaller in absolute value than the zero-tension limit of $\Gamma=-g_{0} / \theta_{0}=-4.2 k_{B} T$.

The force as a function of torque during opening and closing run at the maximal velocity of $2 v=20 \mu \mathrm{m} / \mathrm{s}$ are included in the phase diagram of Fig. 2. These curves follow the ssDNA-dsDNA equilibrium transition, indicating that the opening and the closing of the single base pairs takes place essentially at equilibrium. The relation between torque and force at the moving fork is, under likely experimental conditions, determined by the base-pairing interactions in the same way as at equilibrium. Points A,B,C,D in Fig. 6 and Fig. 7 are mapped to the coexistence curve in Fig. 2; for example, the force peak corresponds to point $\mathrm{B}$ in the phase diagram $f=23 \mathrm{pN}, \Gamma=2.8 k_{B} T$. Turning this 
around, this indicates that the experimental force-displacement curve can be used to infer the torque-displacement curve, using the equilibrium coexistence line of Fig. 2.

The peak forces observed during unzipping are plotted in Fig. 5 (solid line), and match the approximation of the previous section at low forces. Fig. 5 also shows the slightly lower force occurring at the point where the molecule is $50 \%$ unzipped (dot-dashed line). This force is reduced simply because at the half-unzipped point, there is less dsDNA remaining to provide torsional drag than at the peak force point (see Fig. 6).

\section{G. Results for heterogeneous sequence}

Fig. 8 shows the results of numerical integration of (14) using the preaveraged $\lambda$-DNA pairing free energy $g_{0}(n)$ as discussed in Sec. IIIB. The force as a function of the displacement for the velocities $4,8,16,20 \mu \mathrm{m} / \mathrm{s}$ is in good agreement with experimental data, and reflects the sequence; note, for example the progressive increase in $\mathrm{G}-\mathrm{C}$ percentage in the first 2000 base pairs, the decrease from the base pair 20000 to 24000 and the steeper increase from base pair from 24000 to 24500, all these feature are well reproduced in the experimental and theoretical curves. An interesting effect is that the fluctuations in the force due to the sequence are attenuated, especially during rezipping, at higher velocity.

\section{EFFECT OF TWIST RELAXATION}

The initial increase of the force of Fig. 8 is still faster than that observed experimentally. We now examine the effect of the initial twist relaxation dynamics, focusing on its influence on the force signal at the beginning of unzipping. The combined set of equations for the fork and twist comprises a moving-boundary-condition problem that is difficult to solve even by computation. In this section, we construct an approximate solution for the combined twist and opening dynamics, valid when the number of unzipped base pairs remains small with respect to $N$.

\section{A. Memory kernel for fork motion}

We rewrite (9) and (10) as

$$
\tau_{t w} \frac{\partial \theta}{\partial t}=\frac{\partial^{2} \theta}{\partial m^{2}}
$$

where $m$ is the continuous base pair index, and where $\tau_{t w}=4 \pi \eta r^{2} \Delta^{2} / C \simeq 1.410^{-11}$ s. Prior to unzipping, the dsDNA is relaxed,

$$
\theta(m, 0)=0 \quad(0 \leq m \leq N)
$$

The boundary conditions expresses that the $m=N$ extremity of the molecule is free (zero applied torque), while the location and unwinding of the other extremity depend on the number $n(t)$ of unzipped base pairs at time $t$,

$$
\begin{aligned}
& \frac{\partial \theta}{\partial m}(N, t)=0 \\
& \theta(n(t), t)=-\theta_{0} n(t) .
\end{aligned}
$$

We now introduce the Laplace transform of the twist, $\bar{\theta}(m, p)=\int_{0}^{\infty} d t e^{-p t} \theta(m, t)$. The solution of (23) with boundary condition (25) and initial condition (24) reads

$$
\bar{\theta}(m, p)=J(p) \cosh [q(N-m)],
$$

where $q^{2}=p \tau_{t w}$ and $J(p)$ has to be determined to fulfill the remaining boundary condition (26). Defining the Laplace transform $\bar{n}(p)$ of $n(t)$, 26) reads

$$
-\theta_{0} \bar{n}(p)=\int_{0}^{\infty} d t e^{-p t} \theta(n(t), t) \simeq \bar{\theta}(0, p),
$$

as long as $n(t) \ll N$ from (27). Eliminating $J(p)$ from (27) and (28), we obtain 


$$
\bar{\theta}(m, p)=-\theta_{0} \bar{n}(p) \frac{\cosh [q(N-m)]}{\cosh [q N]} .
$$

From (29), the derivative of the twist just upstream of the fork, $\partial \theta / \partial m(n(t), t)$, determines the fork torque. Inserting this into the Laplace transform of the equation of motion (12) for the number of unzipped base pairs yields

$$
\tau_{n} \bar{n}(p)=\frac{1}{p+a q \tanh (q N)} \int_{0}^{\infty} d t e^{-p t}\left[\frac{2 w_{s s}(f)-g_{0}(n(t))}{k_{B} T}\right],
$$

with $a=\theta_{0}^{2} C / \Delta / \tau_{n}$. Inverse Laplace-transforming (30), we obtain a self-consistent integral equation for the number of unzipped base pairs,

$$
n(t)=\int_{0}^{t} d t^{\prime} G\left(t-t^{\prime}\right)\left[\frac{2 w_{s s}(f)-g_{0}(n(t))}{k_{B} T}\right]
$$

where the memory kernel $G$ is defined through

$$
G(\tau)=\frac{1}{\tau_{n}} \int_{-\infty}^{\infty} \frac{d p}{2 \pi i} \frac{e^{p \tau}}{p+a q \tanh (q N)}
$$

Let us stress that the force $f$ in (31) depends on $n$ and $t$ through (7). The poles of (32) are located on the real negative semi-axis, at $p_{\ell}=-\left(y_{\ell} / N\right)^{2} / \tau_{t w} ; y_{\ell}$ is the root (unique) of $\tan y=-b y$ such that $\left|y_{\ell}-\ell \pi\right|<\pi / 2(\ell \geq 0)$, and $b \equiv a N \tau_{t w}$. Calculation of the residues is straightforward, giving

$$
G(\tau)=\Theta(\tau)\left\{\frac{1}{1+b}+2 \sum_{\ell=1}^{\infty} \frac{e^{-\tau / \tau_{\ell}}}{1+b+y_{\ell}^{2} / b}\right\},
$$

where $\Theta(\tau)=1$ if $\tau>0,0$ otherwise. Finally,

$$
\tau_{\ell}=\frac{N^{2}}{y_{\ell}^{2}} \tau_{t w}
$$

gives the $l^{\text {th }}$ elastic relaxation time of the dsDNA region. The longest relaxation time for $\lambda$-DNA is $\tau_{1}=3.510^{-3} \mathrm{~s}$.

If the number of unzipped base pairs $n(t)$ is small with respect to $N$, then (31) is valid. For $\lambda$-DNA this condition happens to be true over the time range $0<t<\tau_{1}$ where $\tau_{1}$ is the longest relaxation mode of the double helix [see (34)]. This means that the force will reach its peak at about $\tau_{1}$, and then will not vary significantly for later times $\sim \tau_{1}$. The dsDNA rotation dynamics reaches a stationary regime, and at later times can be considered to be a rigid cylinder rotating at angular velocity $\omega=\theta_{0} d n / d t$. For times beyond $\tau_{1}$, the twist-relaxation dynamics reduce to just the differential equation for the number of unzipped base pairs (14).

To analyze the dynamics for $t<\tau_{1}$ we solve (31) iteratively. We start from the equilibrium zero speed solution $n_{0}(t) \propto t$. At step $i$, the number of unzipped base pairs as a function of time, $n_{i}(t)$, is inserted in the r.h.s. of (31) and $n_{i+1}(t)$ is collected on the l.h.s. The iteration is repeated until convergence is obtained, which takes about 20 iterations. We also solved the ordinary differential equation (14) using numerical integration routines, and observed that the two curves match accurately for times $t \geq \tau_{1}$ as expected.

\section{B. Results}

Fig. 9a shows force versus displacement including the twist relaxation dynamics for homogeneous sequence $\left(g_{0}=\right.$ $2.5 k_{B} T$ ). The only difference with the curve obtained without the twist propagation (shown for comparison for the velocity of $20 \mu \mathrm{m} / \mathrm{s}$, dashed curve) is the much smoother initial force increase. Theory indicates a force increase spread over the first $\simeq 2.5 \mu \mathrm{m}$ of displacement; after this extension the result converges to the one obtained without twist propagation. While we do observe an initial 'delay' of the force increase, the range over which theory predicts this effect is shorter than that observed experimentally (about $5 \mu \mathrm{m}$ ).

We have also studied the dynamics of the opening fork including both twist propagation and the $\lambda$ sequence. In presence of a complex free energy landscape for $g_{0}$, the iterative scheme exposed above does not converge easily to the solution of (31). We have therefore consider smoother landscapes through a preaveraging of the sequence over $\delta N$ bases, with $\delta N$ ranging from 1000 down to 100 . Such values permit to reach numerical convergence and are sufficient to detect sequence-induced effects on the micron scale. The resulting force signal is shown in Fig. 9b for maximal velocity $2 v=20 \mu \mathrm{m} / \mathrm{s}$. The percentage of GC bases increases during the initial opening, and this effect spreads the initial force increase over the initial $5 \mu \mathrm{m}$ of extension. This trend is in qualitative agreement with experimental findings, though the calculated force is lower than the experimental value by a few $\mathrm{pN}$. 


\section{CONCLUSION}

We have presented a theory of DNA unzipping dynamics, for kilobase or longer dsDNAs. We have shown that torsional drag built by the rotation of the double helix around its axis is the dominant frictional contribution in the opening of $\lambda$-DNA molecules. Easily observable nonequilibrium effects for $\lambda$-DNA are expected for unzipping velocities in excess of $2 \mu \mathrm{m} / \mathrm{s}$. The results of our theory are in agreement with recent observations by Thomen et al [16] of a roughly $40 \%$ increase in unzipping force for $\lambda$-DNA unzipping at $2 v=20 \mu \mathrm{m} / \mathrm{s}$.

We have neglected a few physical effects in the discussion above. First, we have not explicitly included effects of transport of the dsDNA base-pairs to the fork. This is particularly relevant to the experimental setup of Refs. [1,2,16 where the fork moves relative to one of the ssDNA anchor points. This may introduce an additional translational contribution to the dsDNA drag (for a rod model, again proportional to $N-n$ ). However, an estimate made by Thomen et al [16] suggests that this force should be small relative to the unzipping forces. In fact, at the lower fork velocities $\approx 2 \mu \mathrm{m} / \mathrm{s}$ where nonequilibrium effects are observable, separate experiments and theory shows that a $\lambda$-DNA coil should only be slightly stretched [28].

A second factor that we have ignored is the possible effect of dsDNA intrinsic bends. Nelson has recently argued that such bends should induce an orders-of-magnitude effective enhancement in the rotational drag coefficient [29]. In the present experiment, it appears that this effect is nearly absent. Possibly, the roughly twofold enhancement of $r$ over its 'bare' chemical value, needed to generate the observed unzipping force enhancement, is due to permanent bends or other structural inhomogeneities along the rotating double helix.

A third, and potentially interesting effect is that once appreciable torque is built up in the dsDNA, there is the possibility that the upstream dsDNA may begin to writhe 18. For a dsDNA under zero force, writhing (supercoiling) occurs when $|\Gamma|>k_{B} T$ [27]. Tension in excess of $k_{B} T / A \approx 0.1 \mathrm{pN}(A=50 \mathrm{~nm}$ is the dsDNA bending persistence length) pushes the writhing threshold up to $|\Gamma|>\left(4 k_{B} T A f\right)^{1 / 2}$. Writhing (chiral coiling) of the dsDNA could increase the effective $r$, even without the formation of plectonemic supercoils. Formation of plectonemes is straightforward if the dsDNA region starts as a random coil, since there will be near-crossings every few kb (every 5 to 6 persistence lengths) to act as plectoneme 'anchors'. Once plectonemes form, we expect a large enhancement in the effective friction for dsDNA rotation, and a large increase in unzipping force.

It would be very interesting to see results for an experiment carried out in the geometry of Fig. 1 . This might be done using two translated laser traps, which would allow much less perturbation of the dsDNA coil during unzipping.

A natural question is raised by the absence of a noise term in eqn (12), preventing the system from probing the whole free energy landscape at equilibrium [25] (see also [10], Section VII). This approximation, which makes easier for the system to be blocked in a local minimum (stick regime), is expected to be valid at large velocities i.e. when the landscape changes very fast, and times scales are too small to allow for barrier crossing between stick and slip states. To further test the validity of eqn (12), we have calculated the number of open base pairs $n$ as a function of displacement $2 x$ at extremely low velocity e.g. $10 \mathrm{~nm} / \mathrm{s}$ for the $\lambda$ sequence. Results are in very good agreement with equilibrium predictions of Fig. 4; the only difference is that unstick jumps (Inset of Fig. 4) take sometimes place $\leq 5$ nm after their equilibrium counterparts.

Finally, we note that the intrinsic fork motion time $\tau_{n}$ might be larger than the $\approx 10^{-8} / \mathrm{s}$ assumed in this paper using dimensional considerations. The activation barriers to opening of successive bases [7, 12 might make $\tau_{n}$ larger; a recent estimate based on the analysis of a RNA opening experiment at constant force [5] gave $\tau_{n} \simeq 210^{-7} \mathrm{~s}[30]$. However, there will be little consequence of a larger $\tau_{n}$ for the phenomena discussed in this paper, which occur on a much longer time scale.

\section{ACKNOWLEDGEMENTS}

This work was supported by NSF Grant DMR-9734178 and DMR-0203963, by a Research Innovation Award from Research Corporation, and by a Focused Giving Award from Johnson \& Johnson Corporate Research.

[1] U. Bockelmann, B. Essevaz-Roulet, F. Heslot, Proc. Natl. Acad. Sci. USA 94, 11935-40 (1997).

[2] U. Bockelmann, B. Essevaz-Roulet, F. Heslot, Phys. Rev. Lett 79,4489-442 (1997); Phys. Rev. E 58, 2386-2384 (1998).

[3] M. Rief, H. Clausen-Schaumann, H.E. Gaub, Nat. Struct. Biol. 6, 346-9 (1999). 
[4] C. Bustamante, S.B. Smith, J. Liphardt, D. Smith, Curr. Opin. Struct. Biol. 10, 279-85 (2000).

[5] J. Liphardt, B. Oona, S.B. Smith, I. Tinoco, C. Bustamante, Science 292, 733-737 (2001).

[6] J.L. Viovy, C. Heller, F. Caron, P. Cluzel, D. Chatenay, C.R. de l'Acad. des Sci. 317, 795-800 (1994).

[7] R.E. Thompson, E.D. Siggia, Europhys. Lett. 31 335-40 (1995).

[8] K.L. Sebastian, Phys. Rev. E 62 1128-32 (2000).

[9] D.K. Lubensky, D.R. Nelson, Phys. Rev. Lett. 85, 1572-5 (2000).

[10] D.K. Lubensky, D.R. Nelson, Phys. Rev. E 65, 031917 (2002).

[11] S.M. Bhattacharjee, J. Phys. A 33, L423 (2000).

[12] S. Cocco, R. Monasson, J.F. Marko, Proc. Natl. Acad. Sci. USA 98, 8608-13 (2001); S. Cocco, J.F. Marko, R. Monasson, Phys. Rev. E 65, 041907 (2002).

[13] U. Gerland, R. Bundschuh, T. Hwa, Biophys. J. 81, 1324-32 (2001).

[14] T. Strick, J.-F. Allemand, D. Bensimon, R. Lavery, V. Croquette, Physica A 263, 392-404 (1999).

[15] S. Cocco, R. Monasson, Phys. Rev. Lett. 83 5178-81 (1999).

[16] P. Thomen, U. Bockelmann and F. Heslot, Phys. Rev. Lett. 88, 248102 (2002).

[17] C. Levinthal, H.R. Crane, Proc. Natl. Acad. Sci. USA 42, 436 (1956).

[18] J.F. Marko, Phys. Rev. E 57, 2134-49 (1998).

[19] A. Sarkar, J.F. Marko, Phys. Rev. E 64, 1909 (2001).

[20] M. Zuker, Curr. Opin. Struct. Biol 10, 303 (2000).

[21] J. Santa Lucia, Proc. Natl. Acad. Sci. USA 95, 1460-65 (1998).

[22] M. Wang, H. Yin, R. Landick, J. Gelles, S.M. Block, Biophys. J. 72, 1335-1346 (1997).

[23] S.B. Smith, Y. Cui, C. Bustamante, Science 271, 795 (1996).

[24] K.J. Breslauer, R. Frank, H. Blocker, L.A. Marky, Proc. Natl. Acad. Sci. USA 83, 3746-3750 (1986).

[25] U. Bockelmann, P. Thomen, B. Essevaz-Roulet, V. Viasnoff, F. Heslot. Biophys. J. 82, 1537-1553 (2002).

[26] L.D. Landau, E.M. Lifshitz, Theory of Elasticity (Pergamon, New York, 1986), Sec. 16.

[27] J.F. Marko, E.D. Siggia, Phys. Rev. E 52, 2912-2938 (1995).

[28] J.F. Marko, E.D. Siggia, Macromol. 28, 8759 (1995).

[29] P. Nelson, Proc. Natl. Acad. Sci. USA 96, 14342-47 (1999).

[30] S. Cocco, J.F. Marko, R. Monasson, Slow nucleic acid unzipping kinetics from sequence-defined barriers, preprint (2002).

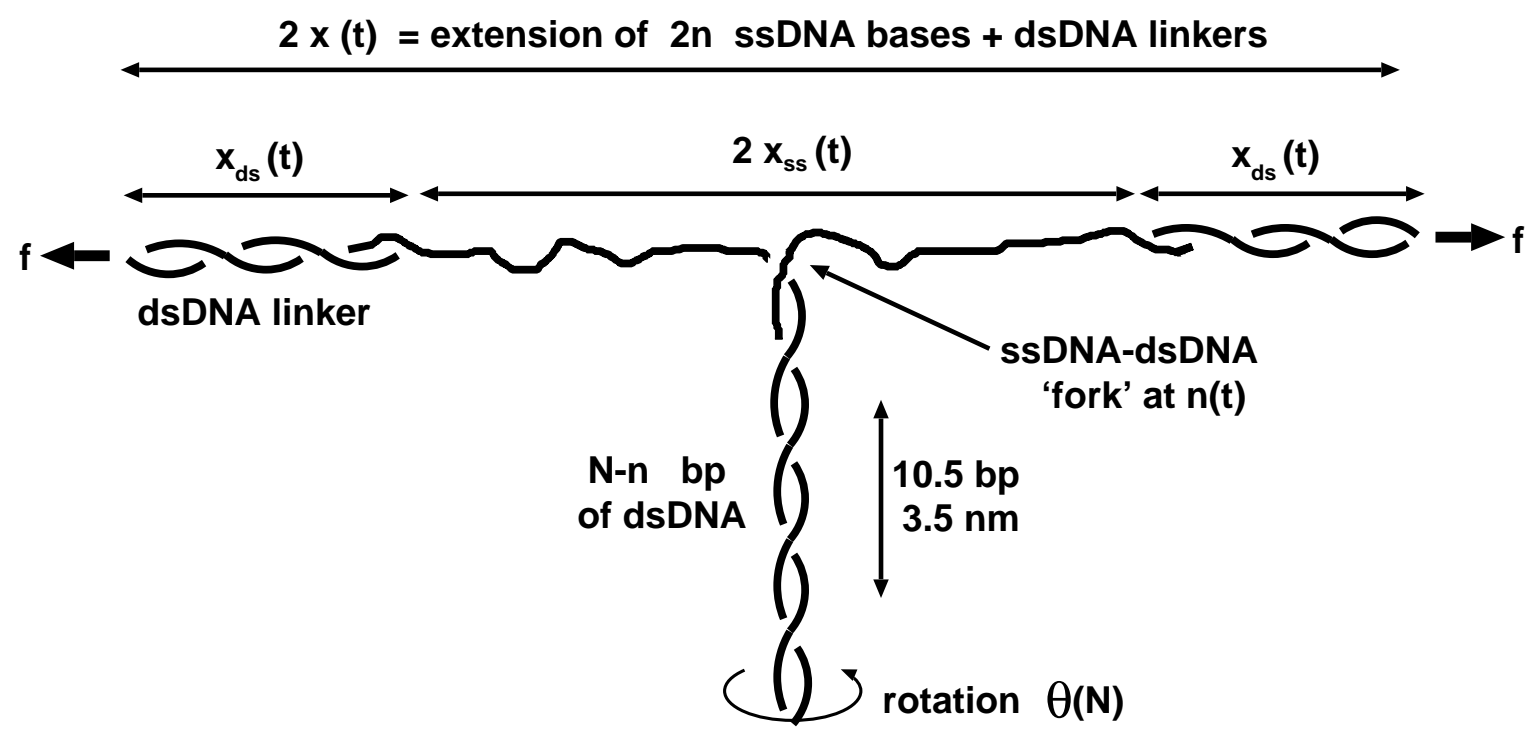

FIG. 1. DNA unzipping experiment considered in this paper. Connections are made to dsDNA linkers attached to the ssDNA ends, and are used to pull adjacent 3' and 5' ssDNA ends apart. The ends of the dsDNA linkers move at equal and opposite velocities of magnitude $v$. As the dsDNA is converted to separated ssDNAs, the helical turns of the dsDNA must be expelled, forcing the remaining dsDNA to be rotated once for each 10.5 bases which are unzipped. 


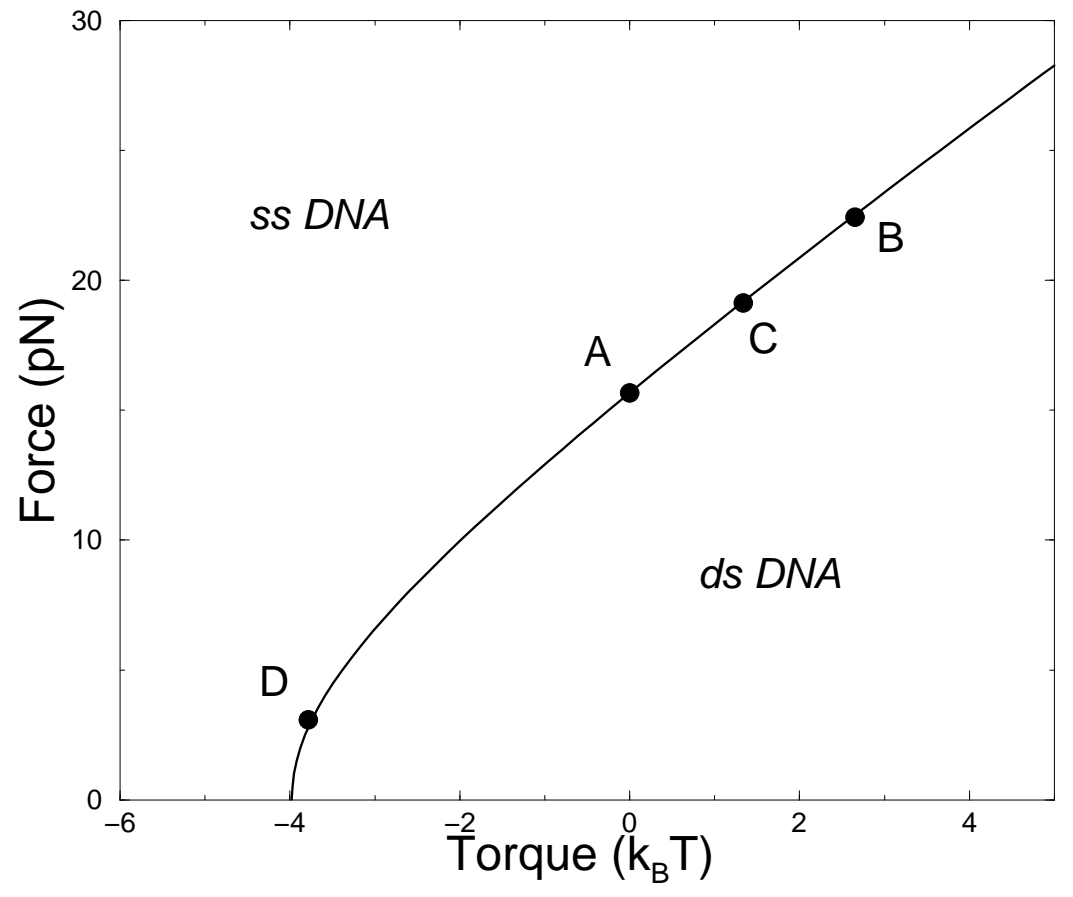

FIG. 2. Phase diagram of a homogeneous DNA molecule under applied force and torque. The base pairing free energy is set to $g_{0}=2.5 k_{B} T$. The double helix conformation is thermodynamically preferred when the applied force is smaller than some torque-dependent critical value, e.g. $f_{u}=15.7 \mathrm{pN}$ at zero torque. Denaturation may be driven by torque only. In the absence of applied force, DNA opens when an underwinding torque larger (in modulus) than $4 k_{B} T$ is applied. Points A,B,C,D refer to the force vs. displacement curve of Fig. 6.

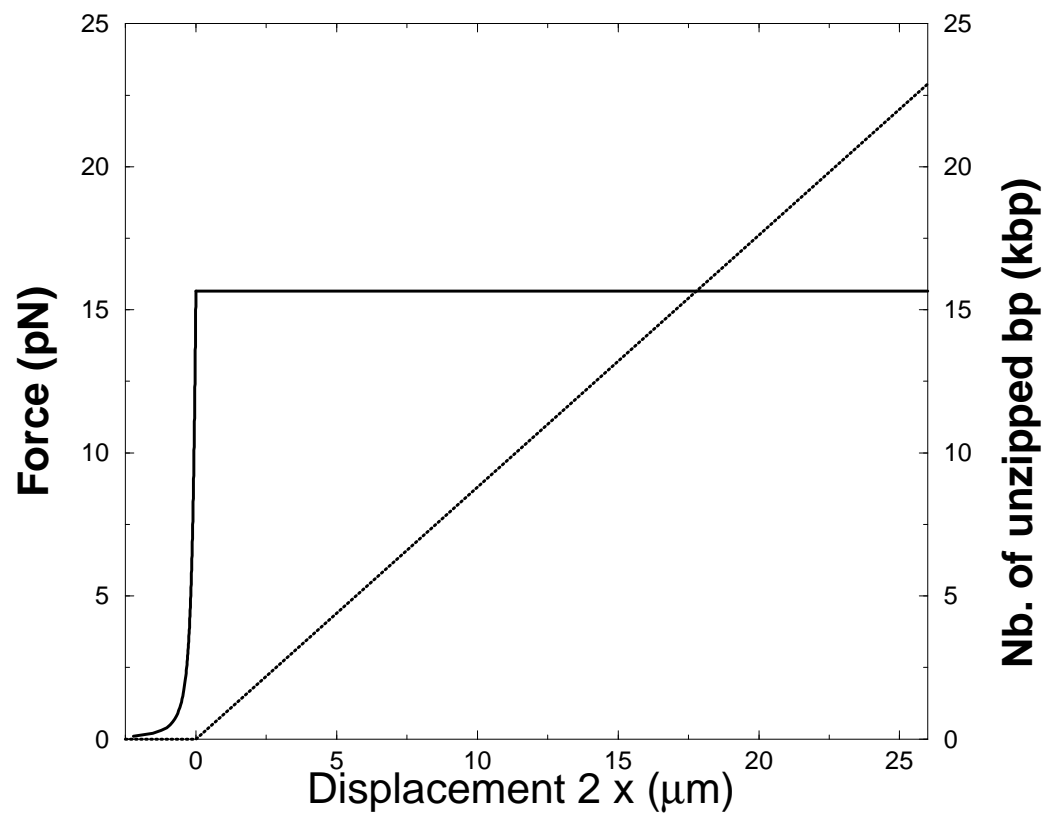

FIG. 3. Force (pN,full curve) and number of unzipped base pairs (kbp, dotted curve) at equilibrium for a homogeneous DNA molecule, as a function of the displacement $x$ after opening initiation. Prior to unzipping $(x<0)$, the force vs. extension curve reflects the elastic behavior of linkers. As opening proceeds $(x>0)$, force is constant at $f_{u}=15.7$ pN, for base pairing energy $g_{0}=2.5 k_{B} T$. The number of unzipped base pairs increases linearly with $x$, with a slope $\simeq 1 \mathrm{bp} / \mathrm{nm}$. 

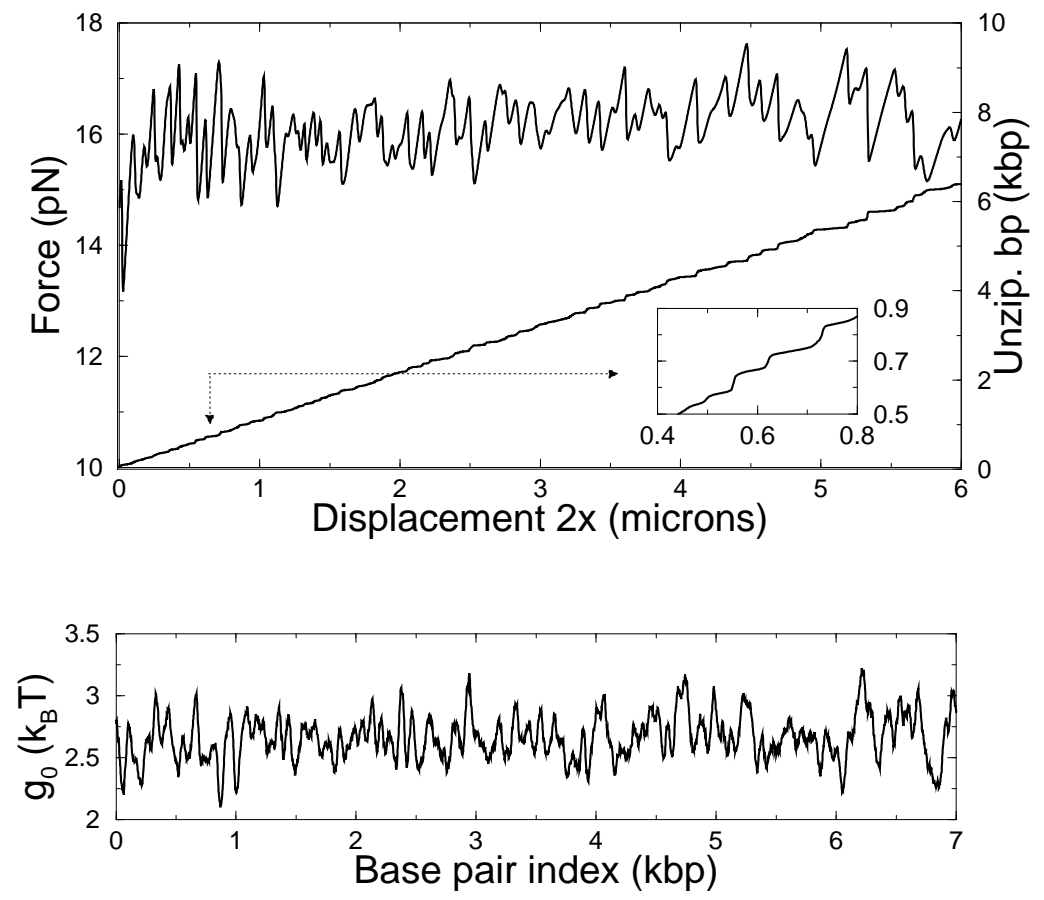

FIG. 4. Force (pN, top curve) and number of unzipped base pairs (kbp, middle curve) at equilibrium for the $\lambda$-DNA molecule, as a function of the displacement $2 x$ after opening initiation. Bottom curve is the base pairing free energy vs. index of base pair, from a Gaussian average over $20 \mathrm{bp}$. The number of unzipped base pairs increases linearly with $x$, with characteristic stick-slip steps (see inset).

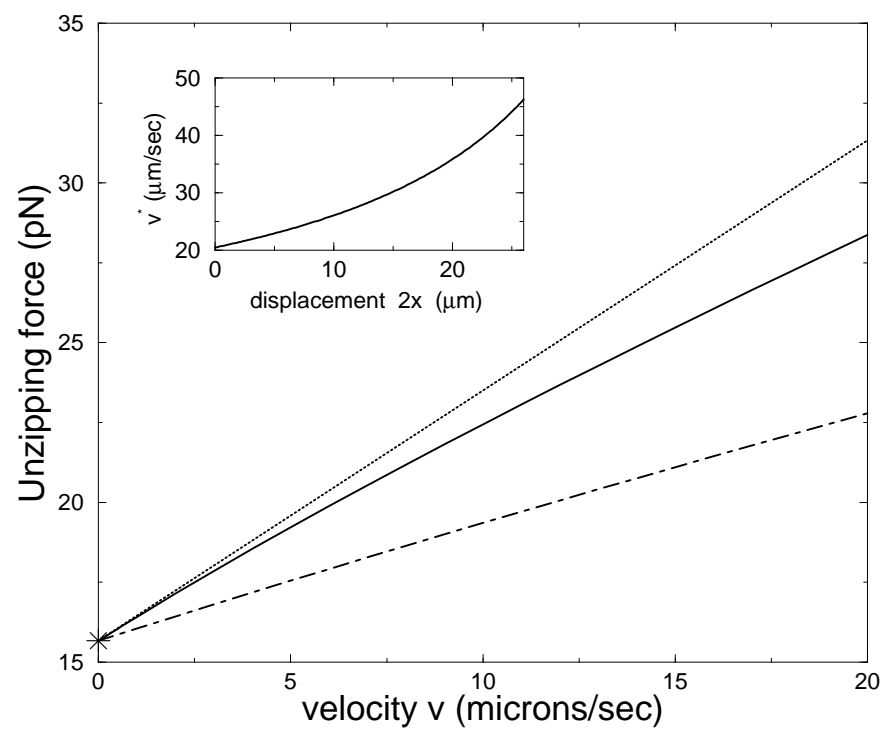

FIG. 5. Theoretical unzipping force as a function of pulling velocity for homogeneous DNA. Star indicates equilibrium unzipping force. Dashed curve shows the 'steady-state' approximate formula (18), solid curve shows the peak force encountered during integration of (14). The dashed and solid curves have the same dependence at low velocity, tending to the static unzipping force $f_{u} \approx 15.7 \mathrm{pN}$ at zero velocity. Inset shows characteristic velocity $v *$ entering steady-state formula is plotted as a function of displacement $2 x$. For velocities above $v^{*}(0)=20 \mu \mathrm{m} / \mathrm{s}$, the steady-state formula starts to be appreciably above the peak force obtained by integration of (14). The dot-dashed curve shows the force at the half-unzipped point when about $25 \mathrm{kbp}$ are unzipped. 


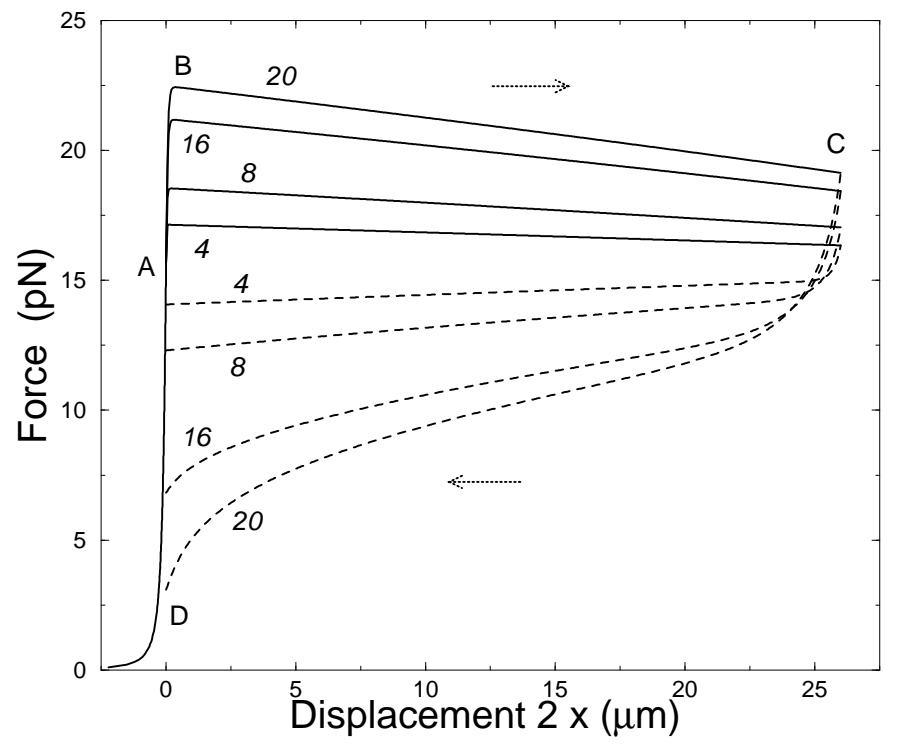

FIG. 6. Force vs. single-strand extension $x=v t$ obtained from integration of (14), for homogeneous DNA plus $7 \mathrm{~kb}$ linkers as in Fig. 1. Outgoing (pulling) curves for $2 v=4,8,16$ and $20 \mu \mathrm{m} / \mathrm{s}$ are shown (solid curves, bottom to top). For these rates, successively higher unzipping forces are obtained. In all cases an initial force increase associated with pulling the linkers taut, is followed by a force peak, and then a slow force reduction. The slow and linear reduction of force with extension is due to progressively less dsDNA being left to provide rotational drag to oppose fork motion. Force during retraction following the extensions are shown for $2 v=4,8,16$ and $20 \mu \mathrm{m} / \mathrm{s}$ (dashed curves). For 2 and $4 \mu \mathrm{m} / \mathrm{s}$, relatively small hysteresis loops occurs. However, at retraction at $\geq 8 \mu \mathrm{m} / \mathrm{s}$ the hysteresis is larger due to larger rotational drag, the force drops near zero at the end of rezipping.

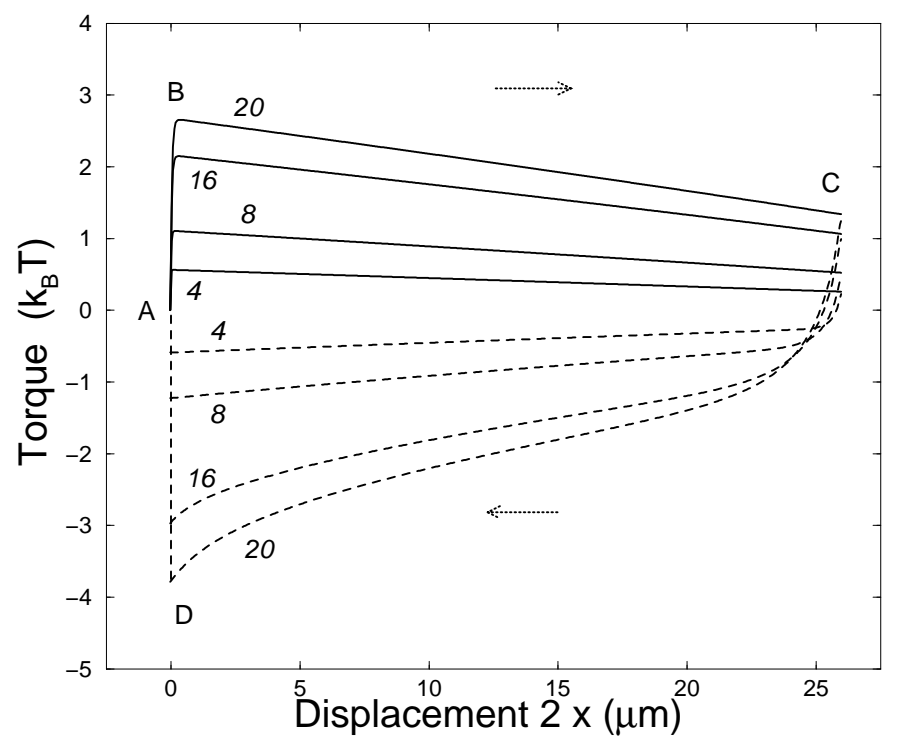

FIG. 7. Torque in the dsDNA region immediately adjacent to the fork, vs single-strand extension $x=v t$, obtained from integration of (14), for the cases shown in Fig. 6. Solid curves indicate extension, dashed retraction. Progressively higher torques are obtained at successively higher pulling velocities. There is an appreciable peak in the torque during the early stages of unzipping, followed by a gradual torque decay as the remaining dsDNA provides progressively less rotational drag. This torque buildup is due to the rapid fork motion forcing the dsDNA region just upstream of the fork to be under overtwisting strain. The torques obtained during retraction (dashed curves) show that rewinding of the molecule generates left-handed elastic torque in the molecule; for retractions of 8 and $16 \mu \mathrm{m} / \mathrm{s}$ this torque exceeds the 'free' limit of $g_{0} / \theta_{0} \approx-4 k_{B} T$ discussed in the text. 


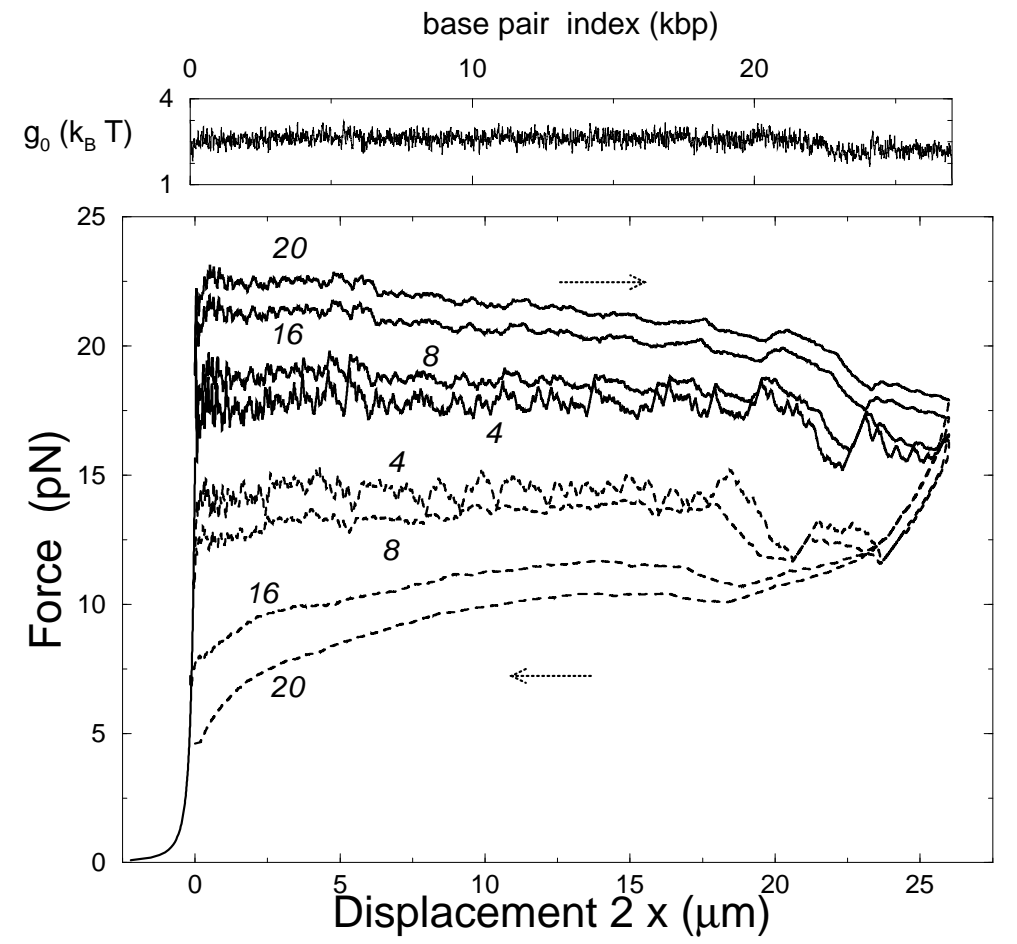

FIG. 8. Force vs. single-strand extension $x=v t$ for $\lambda$-DNA from integration of (14), for $2 v=4,8,16$ and $20 \mu \mathrm{m} / \mathrm{s}$ (outgoing curves: full line, retracting curves: dashed lines). Note that fluctuations of the force due to sequence effects decrease with increasing velocities.
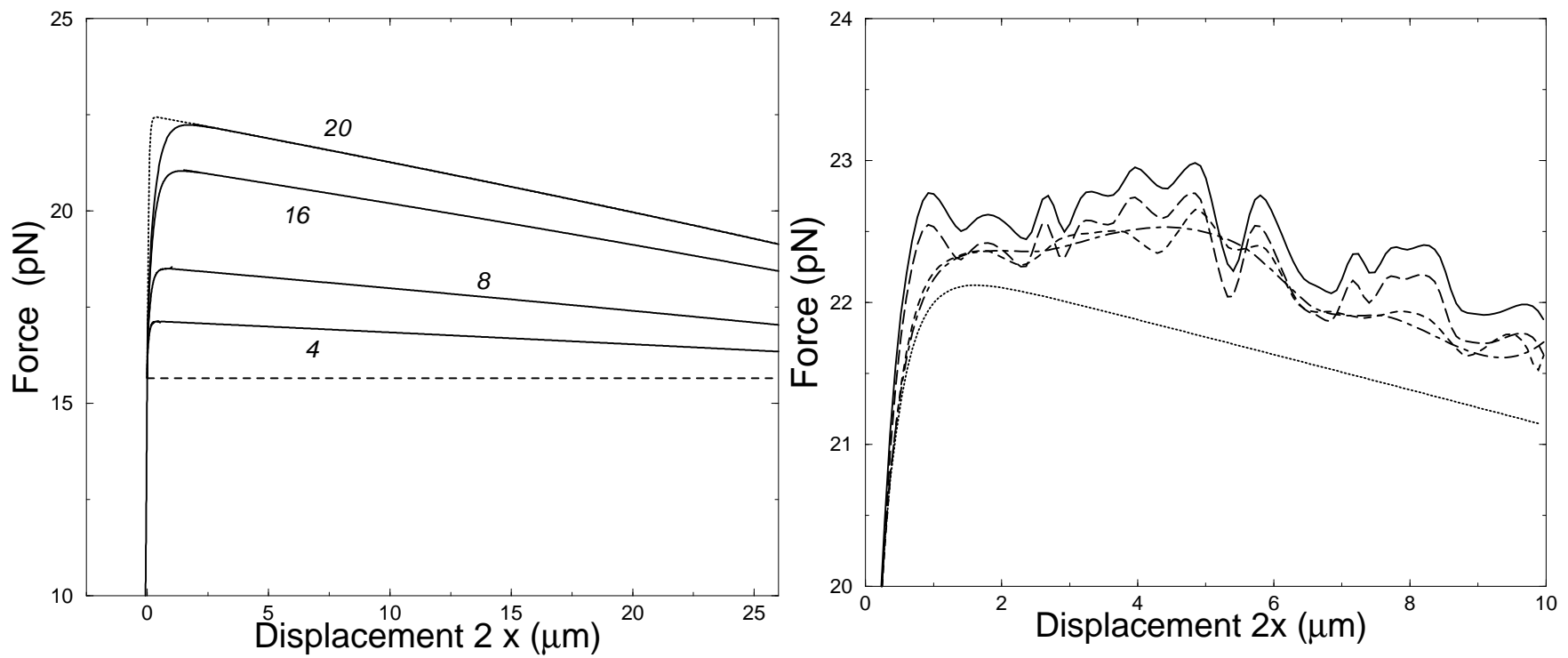

FIG. 9. Force vs. single-strand extension $x=v t$ with twist propagation obtained from resolution of (31). (a) Result for homogeneous DNA $\left(g_{0}=2.5 k_{B} T\right)$ and with $7 \mathrm{~kb}$ linkers. Outgoing (pulling) curves for $2 v=2,4,8,16$ and $20 \mu \mathrm{m} / \mathrm{s}$ are shown (solid curves, bottom to top). For these rates, successively higher unzipping forces are obtained. The initial force increase associated with pulling the linkers is much smoother than without twist propagation (shown for $2 v=20 \mu \mathrm{m} / \mathrm{s}$ ). (b) Corresponding results for inhomogeneous sequence for $2 v=20 \mu \mathrm{m} / \mathrm{s}$, compared to the homogeneous case (dotted line). Curves correspond to preaveraging of the sequence over 1000 (dot-dashed line), 500 (dashed), 250 (long-dashed) and 100 (solid) base pairs. 